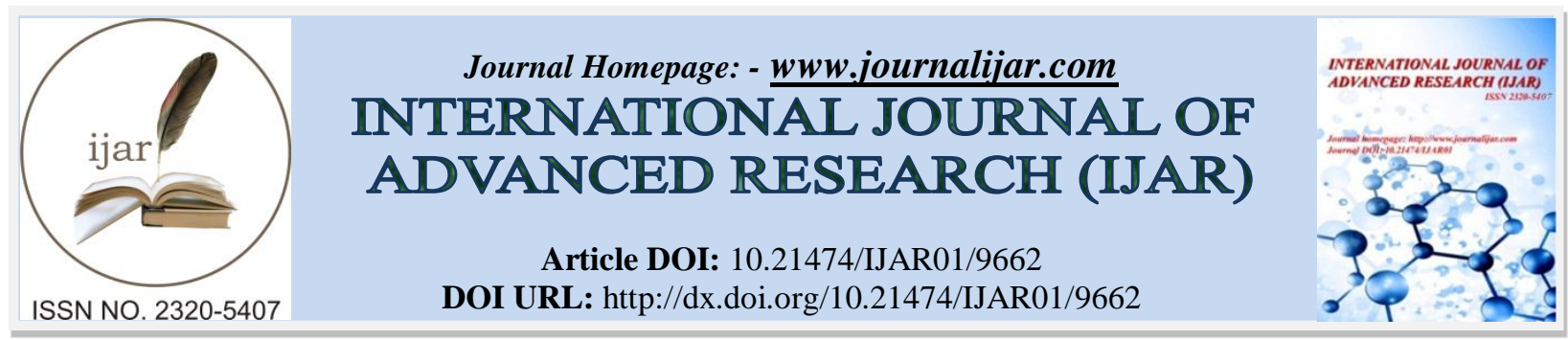

RESEARCH ARTICLE

\title{
STUDY OF DIGITAL MARKETING AND USEFUL TOOLS USED BY SPECIALIST.
}

Mr. Maheshkumar Devendra Mohite.

Research Scholar, Management Consultant, Reside- A/P: Nej, Taluka- Hatkanangale, District- Kolhapur(MH) India.

\section{Manuscript Info}

Manuscript History

Received: 04 July 2019

Final Accepted: 06 August 2019

Published: September 2019

Key words:-

Digital Marketing Tools, Digital

Marketing, Tools of Digital Marketing.

\section{Abstract}

Market demand, winning from computation and accurate result need in business. Digital Marketing activity turns business in very well position. A Specialist used various methods for wins in the market by using technology. The Digital world is complicated. The customer or consumer is smart. Most they are internet users. Recent Technology is trying to reach or catch the maximum customer or consumer. There are various own developed or third party based technique or tools or software used to achieve the accurate target audience. This paper shows that most important digital marketing tools used by the specialist or practitioner in his/ her practice. 50 companies are selected who are special in the digital marketing service provider from Pune city and 500 respondents are taken as sample from selected companies, who are special in digital marketing work. Primary and secondary data was used for data. Reviewed frequently used digital marketing tools and some of them are selected for research study that are Hubspot, Ahrefs, Proof, Survey Anyplace, Yoast, Slack, Trello, Canva Business, Google Adwords, Google Analytics, MailChimp, Asana, BuzzSumo, MeetEdgar, Buffer, Hootsuite, Google Trends, Google Keyword Planner, AppAnnie, Serpstat, SimilarWeb, Facebook Audience Insights, Facebook Ads, Bing Ads, Appstore Search Ads, Manychat, Pushengage, Leanplum, Facebook Analytics, Yandex Metrica, Google Data Studio, Webris SEO Report Tool, Color Picker, Google Analytics URL Builder, Tag Assistant, FB Pixel Helper, Google Tag Manager, Data Scraper, Google Analytics Debugger, Pocket, pCloud Save, Bootstrap Studio, Canva, Flatpack, Marvellapp, Igloo, Adespresso ads, Lapa.ninja, Behnace, Dribbble, Unsplash, Headreach, Buzzstream, Pitchbox, Active Campaign, pCloud, Todoist, Jira, Confluence, Evernote, Sumo.com Blog, Digital marketer, Webris, Neil Patel Blog, Medium, Search Engine Land, Ahrefs Blog, Jon Loomer Blog, Hubspot, Blog, Content Marketing Institute Blog, Buffer Blog, GrooveHQ Blog, MOZ Blog, Adespresso Blog. Convinced Sampling technique was used. Research Shows that digital marketing overview and listing important digital marketing tools which were used by the specialist in his - her work or practice.

Copy Right, IJAR, 2019,. All rights reserved. 


\section{Introduction:-}

Presently Digital Marketing has become an essential part of life. This was used various users for making the valuable benefit in business, it can use by right from personal use to professional use. Digital marketing is a play area for people to keep their lives updated as well as to network with potential clients. It will help their business grow. India is the second largest country. The internet users are over 654 million. These internet users and people are coming to the opportunity for business owners to network with potential customers as well as consumer. This is top reason why Digital Marketing used in India as well as in the world. The customers or consumers are often researching online, offline various info about product or services and then will for buying it. Present customer research into products is particularly popular for confirmable priced items. The Customer or Consumers behaviour is changing day by day. They are always using the Internet to look up product information, compare prices, specification, comments, recommendation and search for deals and promotions. Digital marketing helps to reach target customer or consumer. Costing of marketing is less. Customers or consumers will easy attract towards deal. Digital marketing work did by the human worker using free or paid application software for achieving market. Specialists are using various digital marketing tools for wins in the market.

\section{Objective:-}

1. To understand Digital Marketing

2. To know present useful digital marketing tools used by the specialist or practitioner

\section{Review of Literature}

According to Lucy Alexander (2019) was defined that Digital marketing encompasses all marketing efforts that used an electronic device or the internet. Digital channels were search engines, social media, email, and other websites to connect with current and prospective customers. She was explained that Search Engine Optimization (SEO) is the process of optimizing your website, Content Marketing is generating brand awareness, traffic growth, lead generation, and customers, Social Media Marketing is promoting your brand and your content on social media channels to increase brand awareness, drive traffic, and generate leads for your business. Pay Per Click (PPC) means driving traffic to your or owner website by paying a publisher every time your ads are clicked. Affiliate Marketing is performance-based advertising. Native Advertising can refer to advertisements that were primarily content led and featured on a platform alongside other, non-paid content. Marketing Automation was serving to automate your basic marketing operations. Email Marketing used for communicating with their audiences, Online PR used for securing earned online coverage. Inbound Marketing for attracting, engage, and delight customers at every stage of the buyer's journey.

According to Minos-Athanasios Karyotakis (2019) told that search engine optimization (SEO) practices had emerged in the news media systems. It was spread around the world. He was reviewed literature about the SEO practices by focusing on examining with walkthrough method. He told that news companies allowed the users to comment on their online news articles. He was found that the news websites throughout the globe use similar features and ways to support the comments of the users. In the meantime, though, a high number of the news websites did not allow the users to use their social media accounts in order to comment the provided news articles, or provided multiple comment platforms.

According to Rekha Dahiya \& Gayatri (29 Sep 2017) She was told that maximum automobile industry one of the largest digital spenders. He was studied the effect of digital marketing communication on consumer buying decision process in Indian market. Mixed methodology was adopted for the study. The study concluded that $75 \%$ of the respondents used at least one digital channel of communication for buying a car. Online or Websites was the most used digital channel of communication in buying a car then by 'social networking sites' and 'smartphones.' Buying decision was making process exact from need recognition to post purchase was significantly affected from digital marketing communication with evaluation being the most affected stage. She was confirmed that digital marketing communication is capable in high involvement product category like car. Her results were shown that consumers feel positive towards digital communication.

According to Lindy Tolbert wrote in the article " The Top 16 Best Digital Marketing Tools in 2019" below are important digital marketing tools that are 'Hubspot' can allows you to Grow the traffic, convert leads, and prove ROI for your inbound marketing campaigns and Shorten deal cycles and increase close rates with the provided sales tools. 'Ahrefs' great in competitive analysis, check out who is linking to your competitors, see content ranks, Gap 
tool, identify key weaknesses for your content areas. 'Proof' this tools useful for identifies visitors and examines their journey throughout owner site. 'Survey Anyplace' this was most useful in removing the friction from completing surveys. 'Yoast' this was popular plugin. It works in Gutenberg and the Classic editor in WordPress. It was help you optimize your content for search engines. Slack tools useful for networking and meeting others in the digital marketing space, and gives you the freedom to join or leave channels as needed. Trello is a content management tool. Canva Business useful for creating images using custom pictures, icons, shapes, and fonts. Google Adwords is Google Keyword Planner. Google Analytics was useful for track events to learn more about user engagement. MailChimp was an email marketing and social advertising tool. Asana was record and visualize projects. BuzzSumo is a unique content research tool and another MeetEdgar, Buffer and Hootsuite are useful tools.

Reviews show that present tools are frequently used in company or human worker. Previous Researcher cannot state that which is effective tools, and they hide information about which useful tools used for digital marketing. There are not found sufficient information about digital marketing and tools used for work.

\section{Theoretical background:}

(1) Digital marketing's development did since the Year 1990. (2) Marketing is an activity undertaken by a company or organization or individual or group to promote the buying or selling of a product or service (3) the marketing of products and Services using digital technologies is called as digital marketing. It can be mainly done on the internet but also including phones, display ads or any other digital medium. (4) Digital marketing channels are systems or computer system based, Which is run on the internet; that can create, make, accelerate, and transmit product value from the producer to the terminal consumer by digital networks (5) there are following 'Digital marketing methods' was used that are (a) search engine optimization (SEO), (b) search engine marketing (SEM) (c) content marketing, (d) influencer marketing (e) content automation (f) campaign marketing (g) data-driven marketing (h) viral marketing (i) e-commerce marketing (j) social media marketing (k) social media optimization (l) e-mail direct marketing (m) Display advertising (n) e-books, and optical disks and games (o) mobile phones by SMS and MMS, call-back, and on-hold mobile ring tones. (Reference M D Mohite, investopedia.com, wikipedia.org, books)

\section{Methodology:-}

This research is descriptive in nature. For this study Convenience sampling is used. The area was studied according to the Convenience. Sample Size is 500 employees from selected 50 companies, Research Area: Pune City. Respondents are taken from selected companies, who are special in digital marketing work or they are working in the department of digital marketing from respective companies. Primary data Information is collected through a structured questionnaire and interview. The primary data collected through the structured questionnaire were coded into excel sheet. Likert scale (5 point) used for observe variables. From reviewing frequently used digital marketing tools are selected (74 various tools). Secondary data Information is collected through the reference book, social sites, blogs, articles etc. Collected data analyzed and interpreted by using statistical tools. The data was analyzed and tested using frequency, percentage, Rank and percentage method etc (Project Duration: 10/03/2018 to 10/09/2019)

\section{Data analysis and interpretation:}

Demographic Analysis: Below table (I) shows that response of respondents by Gender \& Below table (II) shows that response of respondents of total Year of Work Experience in Digital Marketing Activity

\begin{tabular}{|l|c|c|c|}
\hline I & Gender & Frequency & Percentage \\
\hline 1 & Male & 250 & $50 \%$ \\
\hline 2 & Female & 250 & $50 \%$ \\
\hline & Total & 500 & $100 \%$ \\
\hline II & Experience & Frequency & Percentage \\
\hline 1 & 0 to 5 Year & 240 & $48 \%$ \\
\hline 2 & 5 to 10 Year & 160 & $32 \%$ \\
\hline 3 & 10 to 15 Year & 88 & $18 \%$ \\
\hline 4 & Above 15 Year & 12 & $2 \%$ \\
\hline & Total & 500 & $100 \%$ \\
\hline
\end{tabular}

Interpretation:

(I) Out of $100 \%$ respondent; male employees were $50 \%$ and Female employees were 50\%. Male \& Female employees are equally responded. (II) Out of $100 \%$ respondent; 0 to 5 year of employees has work experience were 
$48 \%, 5$ to 10 year of year of employees has work experience were $32 \%, 10$ to 15 year of employees has work experience were $18 \%$, Above 15 year of year of employees has work experience were $2 \%$. Majority of employees has 0 to 10 years of employees has work experience. Maximum 0 to 5 Years of experience of employees in digital marketing work.

\section{Responses Analysis:}

Below table 2 shows that response of respondents which were evaluated respondent personally; and they were given opinion about listed tools with the reference of 1: Not important, 2: Less Important, 3: Fifty-Fifty, 4: Important, 5: Very Important is scaling technique and mean score value written.

\begin{tabular}{|c|c|c|c|c|c|c|c|c|}
\hline \multirow[t]{2}{*}{$\begin{array}{c}\text { Sr } \\
\text { No }\end{array}$} & Digital marketing Tools & 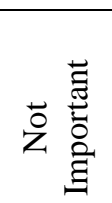 & 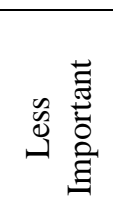 & 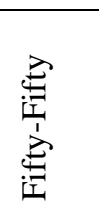 & 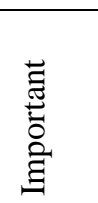 & 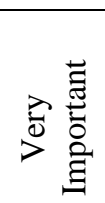 & \multirow[t]{2}{*}{$\begin{array}{c}\text { Total } \\
\%\end{array}$} & \multirow[t]{2}{*}{ Mean } \\
\hline & Scale & 1 & 2 & 3 & 4 & 5 & & \\
\hline \multicolumn{9}{|c|}{ Respondents N = 500 | Responded $100 \%$} \\
\hline 1 & Hubspot & $13 \%$ & $24 \%$ & $25 \%$ & $11 \%$ & $27 \%$ & $100 \%$ & 3.15 \\
\hline 2 & Ahrefs & $11 \%$ & $27 \%$ & $6 \%$ & $49 \%$ & $7 \%$ & $100 \%$ & 3.13 \\
\hline 3 & Proof & $25 \%$ & $16 \%$ & $15 \%$ & $31 \%$ & $13 \%$ & $100 \%$ & 2.93 \\
\hline 4 & Survey Anyplace & $11 \%$ & $29 \%$ & $3 \%$ & $47 \%$ & $10 \%$ & $100 \%$ & 3.17 \\
\hline 5 & Yoast & $6 \%$ & $31 \%$ & $29 \%$ & $31 \%$ & $2 \%$ & $100 \%$ & 2.91 \\
\hline 6 & Slack & $29 \%$ & $7 \%$ & $9 \%$ & $47 \%$ & $9 \%$ & $100 \%$ & 3.00 \\
\hline 7 & Trello & $18 \%$ & $31 \%$ & $8 \%$ & $24 \%$ & $18 \%$ & $100 \%$ & 2.93 \\
\hline 8 & Canva Business & $13 \%$ & $26 \%$ & $28 \%$ & $29 \%$ & $4 \%$ & $100 \%$ & 2.85 \\
\hline 9 & Google Adwords & $10 \%$ & $6 \%$ & $4 \%$ & $51 \%$ & $19 \%$ & $100 \%$ & 3.54 \\
\hline 10 & Google Analytics & $7 \%$ & $24 \%$ & $2 \%$ & $33 \%$ & $34 \%$ & $100 \%$ & 3.64 \\
\hline 11 & MailChimp & $26 \%$ & $25 \%$ & $24 \%$ & $25 \%$ & $0 \%$ & $100 \%$ & 2.48 \\
\hline 12 & Asana & $26 \%$ & $22 \%$ & $20 \%$ & $24 \%$ & $8 \%$ & $100 \%$ & 2.66 \\
\hline 13 & BuzzSumo & $29 \%$ & $31 \%$ & $20 \%$ & $10 \%$ & $9 \%$ & $100 \%$ & 2.39 \\
\hline 14 & MeetEdgar & $28 \%$ & $27 \%$ & $11 \%$ & $26 \%$ & $9 \%$ & $100 \%$ & 2.61 \\
\hline 15 & Buffer & $29 \%$ & $26 \%$ & $29 \%$ & $9 \%$ & $7 \%$ & $100 \%$ & 2.40 \\
\hline 16 & Hootsuite & $25 \%$ & $7 \%$ & $31 \%$ & $29 \%$ & $8 \%$ & $100 \%$ & 2.89 \\
\hline 17 & Google Trends & $16 \%$ & $11 \%$ & $14 \%$ & $47 \%$ & $12 \%$ & $100 \%$ & 3.29 \\
\hline 18 & Google Keyword Planner & $4 \%$ & $28 \%$ & $27 \%$ & $31 \%$ & $10 \%$ & $100 \%$ & 3.14 \\
\hline 19 & AppAnnie & $38 \%$ & $22 \%$ & $24 \%$ & $11 \%$ & $5 \%$ & $100 \%$ & 2.24 \\
\hline 20 & Serpstat & $19 \%$ & $29 \%$ & $25 \%$ & $12 \%$ & $16 \%$ & $100 \%$ & 2.77 \\
\hline 21 & SimilarWeb & $31 \%$ & $31 \%$ & $16 \%$ & $12 \%$ & $10 \%$ & $100 \%$ & 2.38 \\
\hline 22 & Facebook Audience Insights & $15 \%$ & $25 \%$ & $11 \%$ & $31 \%$ & $18 \%$ & $100 \%$ & 3.12 \\
\hline 23 & Facebook Ads & $16 \%$ & $14 \%$ & $10 \%$ & $36 \%$ & $24 \%$ & $100 \%$ & 3.38 \\
\hline 24 & Bing Ads & $11 \%$ & $27 \%$ & $11 \%$ & $36 \%$ & $15 \%$ & $100 \%$ & 3.17 \\
\hline 25 & Appstore Search Ads & $36 \%$ & $4 \%$ & $29 \%$ & $29 \%$ & $2 \%$ & $100 \%$ & 2.57 \\
\hline 26 & Manychat & $25 \%$ & $29 \%$ & $24 \%$ & $11 \%$ & $11 \%$ & $100 \%$ & 2.55 \\
\hline 27 & Pushengage & $25 \%$ & $30 \%$ & $27 \%$ & $10 \%$ & $8 \%$ & $100 \%$ & 2.47 \\
\hline 28 & Leanplum & $13 \%$ & $25 \%$ & $25 \%$ & $11 \%$ & $27 \%$ & $100 \%$ & 3.15 \\
\hline 29 & Facebook Analytics & $11 \%$ & $27 \%$ & $6 \%$ & $49 \%$ & $7 \%$ & $100 \%$ & 3.13 \\
\hline 30 & Yandex Metrica & $26 \%$ & $16 \%$ & $34 \%$ & $11 \%$ & $13 \%$ & $100 \%$ & 2.70 \\
\hline 31 & Google Data Studio & $11 \%$ & $9 \%$ & $23 \%$ & $27 \%$ & $30 \%$ & $100 \%$ & 3.57 \\
\hline 32 & Webris SEO Report Tool & $13 \%$ & $37 \%$ & $24 \%$ & $24 \%$ & $2 \%$ & $100 \%$ & 2.65 \\
\hline 33 & Color Picker & $24 \%$ & $26 \%$ & $34 \%$ & $14 \%$ & $0 \%$ & $100 \%$ & 2.45 \\
\hline 34 & Google Analytics URL Builder & $18 \%$ & $31 \%$ & $8 \%$ & $24 \%$ & $18 \%$ & $100 \%$ & 2.93 \\
\hline 35 & Tag Assistant & $7 \%$ & $24 \%$ & $25 \%$ & $31 \%$ & $14 \%$ & $100 \%$ & 3.20 \\
\hline 36 & FB Pixel Helper & $32 \%$ & $26 \%$ & $22 \%$ & $20 \%$ & $0 \%$ & $100 \%$ & 2.31 \\
\hline
\end{tabular}




\begin{tabular}{|c|c|c|c|c|c|c|c|c|}
\hline 37 & Google Tag Manager & $16 \%$ & $25 \%$ & $15 \%$ & $31 \%$ & $13 \%$ & $100 \%$ & 3.02 \\
\hline 38 & Data Scraper & $31 \%$ & $49 \%$ & $2 \%$ & $7 \%$ & $11 \%$ & $100 \%$ & 2.18 \\
\hline 39 & Google Analytics Debugger & $6 \%$ & $31 \%$ & $29 \%$ & $31 \%$ & $2 \%$ & $100 \%$ & 2.91 \\
\hline 40 & Pocket & $6 \%$ & $29 \%$ & $29 \%$ & $27 \%$ & $8 \%$ & $100 \%$ & 3.02 \\
\hline 41 & pCloud Save & $20 \%$ & $34 \%$ & $4 \%$ & $24 \%$ & $19 \%$ & $100 \%$ & 2.89 \\
\hline 42 & Bootstrap Studio & $9 \%$ & $30 \%$ & $25 \%$ & $27 \%$ & $10 \%$ & $100 \%$ & 2.99 \\
\hline 43 & Canva & $12 \%$ & $26 \%$ & $26 \%$ & $31 \%$ & $5 \%$ & $100 \%$ & 2.92 \\
\hline 44 & Flatpack & $25 \%$ & $34 \%$ & $28 \%$ & $11 \%$ & $2 \%$ & $100 \%$ & 2.31 \\
\hline 45 & Marvellapp & $31 \%$ & $29 \%$ & $23 \%$ & $7 \%$ & $10 \%$ & $100 \%$ & 2.37 \\
\hline 46 & Igloo & $31 \%$ & $31 \%$ & $29 \%$ & $6 \%$ & $2 \%$ & $100 \%$ & 2.17 \\
\hline 47 & Adespresso ads & $22 \%$ & $18 \%$ & $31 \%$ & $20 \%$ & $9 \%$ & $100 \%$ & 2.76 \\
\hline 48 & Lapa.ninja & $31 \%$ & $22 \%$ & $44 \%$ & $2 \%$ & $1 \%$ & $100 \%$ & 2.21 \\
\hline 49 & Behnace & $32 \%$ & $26 \%$ & $40 \%$ & $2 \%$ & $1 \%$ & $100 \%$ & 2.13 \\
\hline 50 & Dribbble & $49 \%$ & $27 \%$ & $20 \%$ & $4 \%$ & $1 \%$ & $100 \%$ & 1.81 \\
\hline 51 & Unsplash & $25 \%$ & $16 \%$ & $15 \%$ & $31 \%$ & $13 \%$ & $100 \%$ & 2.93 \\
\hline 52 & Headreach & $11 \%$ & $29 \%$ & $3 \%$ & $47 \%$ & $10 \%$ & $100 \%$ & 3.17 \\
\hline 53 & Buzzstream & $6 \%$ & $31 \%$ & $29 \%$ & $31 \%$ & $2 \%$ & $100 \%$ & 2.91 \\
\hline 54 & Pitchbox & $29 \%$ & $33 \%$ & $11 \%$ & $20 \%$ & $7 \%$ & $100 \%$ & 2.42 \\
\hline 55 & Active Campaign & $47 \%$ & $26 \%$ & $24 \%$ & $2 \%$ & $1 \%$ & $100 \%$ & 1.84 \\
\hline 56 & pCloud & $27 \%$ & $25 \%$ & $33 \%$ & $11 \%$ & $4 \%$ & $100 \%$ & 2.41 \\
\hline 57 & Todoist & $72 \%$ & $22 \%$ & $0 \%$ & $5 \%$ & $1 \%$ & $100 \%$ & 1.40 \\
\hline 58 & Jira & $48 \%$ & $11 \%$ & $35 \%$ & $4 \%$ & $2 \%$ & $100 \%$ & 1.99 \\
\hline 59 & Confluence & $42 \%$ & $22 \%$ & $34 \%$ & $0 \%$ & $1 \%$ & $100 \%$ & 1.96 \\
\hline 60 & Evernote & $29 \%$ & $31 \%$ & $29 \%$ & $8 \%$ & $2 \%$ & $100 \%$ & 2.23 \\
\hline 61 & Sumo.com Blog & $27 \%$ & $20 \%$ & $24 \%$ & $22 \%$ & $7 \%$ & $100 \%$ & 2.63 \\
\hline 62 & Digital marketer & $28 \%$ & $31 \%$ & $31 \%$ & $4 \%$ & $5 \%$ & $100 \%$ & 2.26 \\
\hline 63 & Webris & $27 \%$ & $25 \%$ & $25 \%$ & $11 \%$ & $13 \%$ & $100 \%$ & 2.58 \\
\hline 64 & Neil Patel Blog & $48 \%$ & $7 \%$ & $26 \%$ & $6 \%$ & $13 \%$ & $100 \%$ & 2.29 \\
\hline 65 & Medium & $9 \%$ & $22 \%$ & $34 \%$ & $18 \%$ & $17 \%$ & $100 \%$ & 3.13 \\
\hline 66 & Search Engine Land & $49 \%$ & $9 \%$ & $3 \%$ & $29 \%$ & $10 \%$ & $100 \%$ & 2.42 \\
\hline 67 & Ahrefs Blog & $25 \%$ & $31 \%$ & $29 \%$ & $10 \%$ & $5 \%$ & $100 \%$ & 2.39 \\
\hline 68 & Jon Loomer Blog & $31 \%$ & $32 \%$ & $27 \%$ & $4 \%$ & $6 \%$ & $100 \%$ & 2.23 \\
\hline 69 & Hubspot Blog & $24 \%$ & $31 \%$ & $17 \%$ & $9 \%$ & $18 \%$ & $100 \%$ & 2.65 \\
\hline 70 & Content Marketing Institute Blog & $27 \%$ & $24 \%$ & $25 \%$ & $11 \%$ & $13 \%$ & $100 \%$ & 2.61 \\
\hline 71 & Buffer Blog & $33 \%$ & $26 \%$ & $26 \%$ & $8 \%$ & $7 \%$ & $100 \%$ & 2.28 \\
\hline 72 & GrooveHQ Blog & $47 \%$ & $22 \%$ & $15 \%$ & $11 \%$ & $5 \%$ & $100 \%$ & 2.05 \\
\hline 73 & MOZ Blog & $25 \%$ & $29 \%$ & $23 \%$ & $13 \%$ & $10 \%$ & $100 \%$ & 2.55 \\
\hline 74 & Adespresso Blog & $21 \%$ & $31 \%$ & $29 \%$ & $18 \%$ & $1 \%$ & $100 \%$ & 2.48 \\
\hline
\end{tabular}

\section{Interpretation:}

$500(100 \%)$ respondents or employees are given response. They were mentioned opinion with reference of selected digital marketing tools \& various importance scales. 'Google Analytics' has highest Mean score is 3.64 and 'Todoist' Lowest mean 1.40 value. According to data analysis Serial number or Item number 57, 50, 66, 58, 64, $55,72,59,19,25,71,49,36,21,46,68,38,45,48,60,13,54,6,15,62,14,56,63,61,70,12,11,30,3,44,51$ and mean score was below 2.93 are 'not important' tools in digital marketing. Serial number or Item number 38, 32, 44, $41,54,68,5,39,46,53,60,67,74,7,13,21,34,62,69,27,42,4,26,40,45,52,73,20,18,2,24,29,50,14,33$, 71,15 and mean score was between 2.18 to 2.40 are 'less important' tools in digital marketing. Serial number or Item number $48,49,58,30,33,59,65,56,16,62,47,25,40,5,15,39,46,53,60,67,74,8,44,27,18,68,43,64$, $71,20,1,2,8,35,42,63,70,11$ and mean score was between 2.21 to 2.48 are 'fifty- fifty important' tools in digital marketing. Serial number or Item number 9, 2, 29, 17, 6, 4, 52, 23, 24, 10, 3, 22, 37, 51, 5, 18, 39, 53, 43, 35, 8, 16, $25,66,42,40,31,14,11,7,34,12,41,32,61,47,54,36$ and mean score was between 2.31 to 3.54 are 'important' tools in digital marketing. Serial number or Item number 10, 31, 1, 28, 23, 9, 41, 7, 34, 69, 22, 65, 20, 24, 35, 70, 3, 
$30,37,51,63,64,17,26,38,4,45,52,66,73,21,18,42,13,6,14,47,40$ and mean score was between 3.02 to 3.64 are 'very important' tools in digital marketing.

Ranking and Percentage:

Below table 3 shows Ranking and Percentage of Useful Digital Marketing Tools used by specialist or practitioner.

\begin{tabular}{|c|c|c|c|c|}
\hline $\mathrm{Sr}$ & Digital marketing Tools & Mean & Rank & Percentage \\
\hline 10 & Google Analytics & 3.64 & 1 & $100.00 \%$ \\
\hline 31 & Google Data Studio & 3.57 & 2 & $98.60 \%$ \\
\hline 9 & Google Adwords & 3.54 & 3 & $97.20 \%$ \\
\hline 23 & Facebook Ads & 3.38 & 4 & $95.80 \%$ \\
\hline 17 & Google Trends & 3.29 & 5 & $94.50 \%$ \\
\hline 35 & Tag Assistant & 3.20 & 6 & $93.10 \%$ \\
\hline 24 & Bing Ads & 3.17 & 7 & $91.70 \%$ \\
\hline 4 & Survey Anyplace & 3.17 & 8 & $89.00 \%$ \\
\hline 52 & Headreach & 3.17 & 8 & $89.00 \%$ \\
\hline $\mathbf{1}$ & Hubspot & 3.15 & 10 & $86.30 \%$ \\
\hline 28 & Leanplum & 3.15 & 10 & $86.30 \%$ \\
\hline 18 & Google Keyword Planner & 3.14 & 12 & $84.90 \%$ \\
\hline $\mathbf{2}$ & Ahrefs & 3.13 & 13 & $82.10 \%$ \\
\hline 29 & Facebook Analytics & 3.13 & 13 & $82.10 \%$ \\
\hline 65 & Medium & 3.13 & 15 & $80.80 \%$ \\
\hline 22 & Facebook Audience Insights & 3.12 & 16 & $\mathbf{7 9 . 4 0 \%}$ \\
\hline 40 & Pocket & 3.02 & 17 & $\mathbf{7 8 . 0 0 \%}$ \\
\hline 37 & Google Tag Manager & 3.02 & 18 & $\mathbf{7 6 . 7 0 \%}$ \\
\hline 6 & Slack & 3.00 & 19 & $75.30 \%$ \\
\hline 42 & Bootstrap Studio & 2.99 & 20 & $\mathbf{7 3 . 9 0 \%}$ \\
\hline 7 & Trello & 2.93 & 21 & $71.20 \%$ \\
\hline 34 & Google Analytics URL Builder & 2.93 & 21 & $71.20 \%$ \\
\hline 3 & Proof & 2.93 & 23 & $68.40 \%$ \\
\hline 51 & Unsplash & 2.93 & 23 & $68.40 \%$ \\
\hline 43 & Canva & 2.92 & 25 & $67.10 \%$ \\
\hline 5 & Yoast & 2.91 & 26 & $63.00 \%$ \\
\hline 39 & Google Analytics Debugger & 2.91 & 26 & 63.00\% \\
\hline 53 & Buzzstream & 2.91 & 26 & $63.00 \%$ \\
\hline 16 & Hootsuite & 2.89 & 29 & $61.60 \%$ \\
\hline 41 & pCloud Save & 2.89 & 30 & $60.20 \%$ \\
\hline 8 & Canva Business & 2.85 & 31 & $\mathbf{5 8 . 9 0 \%}$ \\
\hline 20 & Serpstat & 2.77 & 32 & $\mathbf{5 7 . 5 0 \%}$ \\
\hline 47 & Adespresso ads & 2.76 & 33 & $56.10 \%$ \\
\hline 30 & Yandex Metrica & 2.70 & 34 & $54.70 \%$ \\
\hline 12 & Asana & 2.66 & 35 & $\mathbf{5 3 . 4 0 \%}$ \\
\hline 32 & Webris SEO Report Tool & 2.65 & 36 & $50.60 \%$ \\
\hline 69 & Hubspot Blog & 2.65 & 36 & $\mathbf{5 0 . 6 0 \%}$ \\
\hline 61 & Sumo.com Blog & 2.63 & 38 & $49.30 \%$ \\
\hline 14 & MeetEdgar & 2.61 & 39 & $46.50 \%$ \\
\hline 70 & Content Marketing Institute Blog & 2.61 & 39 & $46.50 \%$ \\
\hline 63 & Webris & 2.58 & 41 & $45.20 \%$ \\
\hline 25 & Appstore Search Ads & 2.57 & 42 & $43.80 \%$ \\
\hline 73 & MOZ Blog & 2.55 & 43 & $42.40 \%$ \\
\hline 26 & Manychat & 2.55 & 44 & $41.00 \%$ \\
\hline 11 & MailChimp & 2.48 & 45 & $39.70 \%$ \\
\hline 74 & Adespresso Blog & 2.48 & 46 & $38.30 \%$ \\
\hline 27 & Pushengage & 2.47 & 47 & $36.90 \%$ \\
\hline 33 & Color Picker & 2.45 & 48 & $35.60 \%$ \\
\hline
\end{tabular}




\begin{tabular}{|r|l|r|r|r|}
\hline $\mathbf{6 6}$ & Search Engine Land & $\mathbf{2 . 4 2}$ & $\mathbf{4 9}$ & $\mathbf{3 4 . 2 0 \%}$ \\
\hline $\mathbf{5 4}$ & Pitchbox & $\mathbf{2 . 4 2}$ & $\mathbf{5 0}$ & $\mathbf{3 2 . 8 0 \%}$ \\
\hline $\mathbf{5 6}$ & pCloud & $\mathbf{2 . 4 1}$ & $\mathbf{5 1}$ & $\mathbf{3 1 . 5 0 \%}$ \\
\hline $\mathbf{1 5}$ & Buffer & $\mathbf{2 . 4 0}$ & $\mathbf{5 2}$ & $\mathbf{3 0 . 1 0 \%}$ \\
\hline $\mathbf{6 7}$ & Ahrefs Blog & $\mathbf{2 . 3 9}$ & $\mathbf{5 3}$ & $\mathbf{2 8 . 7 0 \%}$ \\
\hline $\mathbf{1 3}$ & BuzzSumo & $\mathbf{2 . 3 9}$ & $\mathbf{5 4}$ & $\mathbf{2 7 . 3 0 \%}$ \\
\hline $\mathbf{2 1}$ & SimilarWeb & $\mathbf{2 . 3 8}$ & $\mathbf{5 5}$ & $\mathbf{2 6 . 0 0 \%}$ \\
\hline $\mathbf{4 5}$ & Marvellapp & $\mathbf{2 . 3 7}$ & $\mathbf{5 6}$ & $\mathbf{2 4 . 6 0 \%}$ \\
\hline $\mathbf{3 6}$ & GB Pixel Helper & $\mathbf{2 . 3 1}$ & $\mathbf{5 7}$ & $\mathbf{2 3 . 2 0 \%}$ \\
\hline $\mathbf{4 4}$ & Flatpack & $\mathbf{2 . 3 1}$ & $\mathbf{5 8}$ & $\mathbf{2 1 . 9 0 \%}$ \\
\hline $\mathbf{6 4}$ & Neil Patel Blog & $\mathbf{2 . 2 9}$ & $\mathbf{5 9}$ & $\mathbf{2 0 . 5 0 \%}$ \\
\hline $\mathbf{7 1}$ & Buffer Blog & $\mathbf{2 . 2 8}$ & $\mathbf{6 0}$ & $\mathbf{1 9 . 1 0 \%}$ \\
\hline $\mathbf{6 2}$ & Digital marketer & $\mathbf{2 . 2 6}$ & $\mathbf{6 1}$ & $\mathbf{1 7 . 8 0 \%}$ \\
\hline 19 & AppAnnie & $\mathbf{2 . 2 4}$ & $\mathbf{6 2}$ & $\mathbf{1 6 . 4 0 \%}$ \\
\hline $\mathbf{6 8}$ & Jon Loomer Blog & $\mathbf{2 . 2 3}$ & $\mathbf{6 3}$ & $\mathbf{1 5 . 0 0 \%}$ \\
\hline $\mathbf{6 0}$ & Evernote & $\mathbf{2 . 2 3}$ & $\mathbf{6 4}$ & $\mathbf{1 3 . 6 0 \%}$ \\
\hline $\mathbf{4 8}$ & Lapa.ninja & $\mathbf{2 . 2 1}$ & $\mathbf{6 5}$ & $\mathbf{1 2 . 3 0 \%}$ \\
\hline 38 & Data Scraper & $\mathbf{2 . 1 8}$ & $\mathbf{6 6}$ & $\mathbf{1 0 . 9 0 \%}$ \\
\hline $\mathbf{4 6}$ & Igloo & $\mathbf{2 . 1 7}$ & $\mathbf{6 7}$ & $\mathbf{9 . 5 0 \%}$ \\
\hline 49 & Behnace & $\mathbf{2 . 1 3}$ & $\mathbf{6 8}$ & $\mathbf{8 . 2 0 \%}$ \\
\hline $\mathbf{7 2}$ & GrooveHQ Blog & $\mathbf{2 . 0 5}$ & $\mathbf{6 9}$ & $\mathbf{6 . 8 0 \%}$ \\
\hline $\mathbf{5 8}$ & Jira & $\mathbf{1 . 9 9}$ & $\mathbf{7 0}$ & $\mathbf{5 . 4 0 \%}$ \\
\hline $\mathbf{5 9}$ & Confluence & $\mathbf{1 . 9 6}$ & $\mathbf{7 1}$ & $\mathbf{4 . 1 0 \%}$ \\
\hline $\mathbf{5 5}$ & Active Campaign & $\mathbf{1 . 8 4}$ & $\mathbf{7 2}$ & $\mathbf{2 . 7 0 \%}$ \\
\hline $\mathbf{5 0}$ & Dribbble & $\mathbf{1 . 8 1}$ & $\mathbf{7 3}$ & $\mathbf{1 . 3 0 \%}$ \\
\hline $\mathbf{5 7}$ & Todoist & $\mathbf{1 . 4 0}$ & $\mathbf{7 4}$ & $\mathbf{0 . 0 0 \%}$ \\
\hline & & & &
\end{tabular}

\section{Interpretation:}

With reference of ranking and percentage table it seen that top 74 tools which are sorted by highest mean score to lowest mean score and provide rank to respective tools. Ranking $1^{\text {st }}$ to $74^{\text {th }}$ given by analysis. It was seen that between $75 \%$ to $100 \%$ and Mean score between 3.00 to 3.64 are found in rank 1 to 19 which of them tools are Google Analytics, Google Data Studio, Google Adwords, Facebook Ads, Google Trends, Tag Assistant, Bing Ads, Survey Anyplace, Headreach, Hubspot, Leanplum, Google Keyword Planner, Ahrefs, Facebook Analytics, Medium, Facebook Audience Insights, Pocket, Google Tag Manager, Slack useful digital marketing tools. It was seen that between $50 \%$ to $75 \%$ and Mean score between 2.65 to 2.99 are found in rank 20 to 36 which of them tools are Bootstrap Studio, Trello, Google Analytics URL Builder, Proof, Unsplash, Canva, Yoast, Google Analytics Debugger, Buzzstream, Hootsuite, pCloud Save, Canva Business, Serpstat, Adespresso ads, Yandex Metrica, Asana, Webris SEO Report Tool, Hubspot Blog. It was seen that between $25 \%$ to 50\% and Mean score between 2.38 to 2.63 are found in rank 38 to 55 which of them tools are Sumo.com Blog, MeetEdgar, Content Marketing Institute Blog, Webris, Appstore Search Ads, MOZ Blog, Manychat, MailChimp, Adespresso Blog, Pushengage, Color Picker, Search Engine Land, Pitchbox, pCloud, Buffer, Ahrefs Blog, BuzzSumo, SimilarWeb. It was seen that between $00 \%$ to $25 \%$ and Mean score between 1.40 to 2.37 are found in rank 56 to 74 which of them tools are Marvellapp, FB Pixel Helper, Flatpack, Neil Patel Blog, Buffer Blog, Digital marketer, AppAnnie, Jon Loomer Blog, Evernote, Lapa.ninja, Data Scraper, Igloo, Behnace, GrooveHQ Blog, Jira, Confluence, Active Campaign, Dribbble, Todoist.

Majority of respondent are more confident with or told that more useful tools are as Google Analytics has Rank 1, Google Data Studio has Rank 2, Google Adwords has Rank 3, Facebook Ads has Rank 4, Google Trends has Rank 5, Tag Assistant has Rank 6, Bing Ads has Rank 7, Survey Anyplace has Rank 8, Headreach has

Rank 8, Hubspot has Rank 10, Leanplum has Rank 10, Google Keyword Planner has Rank 12, Ahrefs has Rank 13, Facebook Analytics has Rank 13, Medium has Rank 15, Facebook Audience Insights has Rank 16, Pocket has Rank 17, Google Tag Manager has Rank 18, Slack has Rank 19, Bootstrap Studio has Rank 20, Trello has Rank 21, Google Analytics URL Builder has Rank 21, Proof has Rank 23, Unsplash has Rank 23, Canva has Rank 25, Yoast has Rank 26, Google Analytics Debugger has Rank 26, Buzzstream has Rank 26, Hootsuite has Rank 29, pCloud Save has Rank 30, Canva Business has Rank 31, Serpstat has Rank 32, 
Adespresso ads has Rank 33, Yandex Metrica has Rank 34, Asana has Rank 35, Webris SEO Report Tool has Rank 36, Hubspot Blog has Rank 36.

\section{Finding}

The specialist used various techniques for marketing. Each and every digital marketing tool has own specification and benefit. According to study there are selected 74 tools are important for any digital marketing projects. A researcher found that there are only 37 tools are useful for digital marketing work. Most respondents told that paid tools are more benefited than free tools.

\section{Suggestion}

(1) There are important for marketing operations is establishing a project workflow between marketing and the rest of the organization or companies. (2) Read mind of the customer or consumer or the audience. Monitor and collect information of Customer (his/ her) interest. (3) Use, implement and develop special technique with the help of digital marketing tools for marketing. (4) Secure customer data, digital marketing work plan and be safe from external threats. (5) stay honest with the customer. (6) Study deeply with third party tools.

\section{Conclusion:-}

Present business environment trust on digital marketing and its techniques. They are relying on digital marketing for best result in future. Specialist told that Digital marketing reduced cost as comparing to traditional marketing. Specialist used various paid or free third party application software to win in market. Researcher was studied 74 digital marketing tools which are useful and has its own advantages during work. Research study shows that there are 37 digital marketing tools are more useful for work. These are Google Analytics, Google Data Studio, Google Adwords, Facebook Ads, Google Trends, Tag Assistant, Bing Ads, Survey Anyplace, Headreach, Hubspot, Leanplum, Google Keyword Planner, Ahrefs, Facebook Analytics, Medium, Facebook Audience Insights, Pocket, Google Tag Manager, Slack, Bootstrap Studio, Trello, Google Analytics URL Builder, Proof, Unsplash, Canva, Yoast, Google Analytics Debugger, Buzzstream, Hootsuite, pCloud Save, Canva Business, Serpstat, Adespresso ads, Yandex Metrica, Asana, Webris SEO Report Tool, Hubspot Blog. Digital Marketing is easy way to promote the buying or selling of product or service.

\section{Acknowledgement:-}

"Study was understood overview of digital marketing and useful digital marketing tools used by specialist. There are requiring implementation of new modern technique, process and concept in Digital Marketing. Effective, user friendly and cheap Digital marketing tools require in modern business". \{Thanks to Volunteer Person name Mr. Yuvraj Kamble, B.E. as Assistant (from Institute: STBCET, Tuljapur M 9867555699), Miss Pooja Bargale, M.Phil as Assistant (from Institute: CSIBER, Kolhapur) whose were Successfully Did Assigned Responsibility for Completion of this Research | Title of Project: "Study of Digital Marketing and Useful Tools Used by Specialist" Project Duration 10/03/2018 to 10/09/2019\}.

\section{Reference:-}

1. Lucy Alexander (2019), 'What Is Digital Marketing', published in blog.hubspot.com/marketing/what-is-digitalmarketing

2. Minos-Athanasios Karyotakis (2019) “Search Engine Optimization Practices: A Study about the Way News Websites Allow the Users to Comment on Their News Articles" published in academia.edu/Documents/in/Digital_Marketing

3. Rekha Dahiya \& Gayatri (29 Sep 2017) "A Research Paper on Digital Marketing Communication and Consumer Buying Decision Process: An Empirical Study in the Indian Passenger Car Market” Journal of Global Marketing , p 73 Volume 31, $2018 \quad-$ Issue 2: Marketing Challenges and Opportunities in India doi.org/10.1080/08911762.2017.1365991

4. Lindy Tolbert (2019) “ The Top 16 Best Digital Marketing Tools in 2019" published in blog.hubspot.com/marketing/digital-marketing-tools

5. Kothari C. R. ,(2004), "Research Methodology, Methods and techniques" (2nd ed.), New Delhi: New age International (p) Ltd.

6. Harry N Boone, Deborah A Boone, (2012),"'analyzing likert data", journal of extension, vol 50.

7. Geoff Norman, (2010), "Likert scales, levels of measurement and the laws of statistics", Springer Science Business Media B.V.

8. Maheshkumarmohite.com, blogs, comments, web-portal, researchgate.com, wekipedia. 